\title{
"SPECIAL" FAMILIES AND THEIR "NORMAL" DAILY LIVES: FAMILY QUALITY OF LIFE AND THE SOCIAL ENVIRONMENT
}

\author{
Femke Boelsma, Alice Schippers, Menco Dane, and Tineke A. Abma
}

\begin{abstract}
This article focuses on the social and societal factors that influence family quality of life (FQOL). In this qualitative study from the Netherlands, a multiple case study design was used, in which members of families having a child or children with intellectual and developmental disabilities talked about their experiences during interviews. The data were analysed through a rigorous inductive thematic analysis. Our findings relate to the families' experiences with their social environment and especially with the support they received. We argue that processes of acceptance and understanding play a role in FQOL. Also, we illustrate how the family members experience their interaction with the community, and how moral norms and values could influence the lives of the families. The families reflected on notions of normality and being different, and sometimes struggled with the implicit norms and values of society, which can impede acceptance and understanding by others. In turn, these norms and values influence the level of support from other people and the possibilities for social interaction with the community.
\end{abstract}

Keywords: family, quality of life, qualitative research, social environment, acceptance, social inclusion

Femke Boelsma MSc (the corresponding author) is a researcher at the Department of Youth \& Lifestyle, Institute of Health Sciences, Free University Amsterdam, De Boelelaan 1085, 1081 HV Amsterdam, The Netherlands. Email: f.boelsma@vu.nl

Alice Schippers PhD is General Director of the Disability Studies in the Netherlands Foundation and senior researcher at the Department of Medical Humanities, Amsterdam UMC, Free University Amsterdam, Amsterdam Public Health Research Institute, De Boelelaan 1117, 1081 HV, Amsterdam, The Netherlands. Email: alice.schippers@disabilitystudies.nl

Menco Dane MSc is a research partner at the Department of Medical Humanities, Amsterdam UMC, Free University Amsterdam, Amsterdam Public Health Research Institute, De Boelelaan 1117, 1081 HV, Amsterdam, The Netherlands. Email: mrdane@xs4all.nl

Tineke A. Abma $\mathrm{PhD}$ is Professor of Participation \& Diversity and Research Director at the Department of Medical Humanities, Amsterdam UMC, Free University Amsterdam, Amsterdam Public Health Research Institute, De Boelelaan 1117, 1081 HV, Amsterdam, The Netherlands. Email: t.abma@vumc.nl 
International Journal of Child, Youth and Family Studies (2018) 9(4): 107-124

Family quality of life (FQOL) is an emerging concept that is used to understand and improve the well-being and quality of life (QOL) of families. FQOL originated from the concept of individual QOL which incorporates both subjective and objective components and is a multidimensional concept that reflects the perceptions of a person on their life (Schalock et al., 2002). Individual QOL domains include: emotional well-being, interpersonal relations, material well-being, personal development, physical well-being, self-determination, social inclusion, and rights (IASSID SIRG-QOL, 2000; Schalock et al., 2002). Four more domains were added to form the concept of FQOL, which are: daily family life, parenting, family interactions, and financial well-being (Poston et al., 2003). In the FQOL field, two main FQOL instruments have been developed: the Beach Center FQOL Scale (Beach Center on Disability, 2005; Hoffman, Marquis, Poston, Summers, \& Turnbull, 2006) and the FQOL Survey-2006 (Brown et al., 2006). Zuna, I. Brown, and R. I. Brown (2014) defined FQOL as:

For the most part, families consider their lives to be happy and fulfilling if all members are healthy, have a safe place to live, have a stable income, enjoy their lives together, have opportunities to learn and improve, benefit from their community supports and resources, and experience fulfilling social relationships with others. (p. 162)

Most research in the FQOL field has focused on families with one or more children with an intellectual or developmental disability (ID/DD), because families are increasingly fulfilling a central role in the support and care of their children with ID/DD (Brown et al., 2006). The Beach Center FQOL Scale and the FQOL Survey-2006 include questions relating to the support within a family, but also rate the importance, satisfaction, and attainment of support from other people. Results from various countries of the FQOL-2006 indicate that while support from other people is rated as being important, it scores low on satisfaction in almost all countries (Brown, Kyrkou, \& Samuel, 2016). A study in Australia found that social support from others is needed in order to be included in the community and it contributes to the resilience and good health of family members. At the same time, families indicate that it is difficult to acquire social support from others (Faragher, Broadbent, Brown, \& Burgess, 2014). A study by Brown, Anand, Fung, Isaacs, and Baum (2003) reported that this is partly due to urban lifestyle, which often provides few opportunities for social support. Also, some families stated that they did not want to ask other peoples' help because they had found other people unable to relate to them, which contributed to their feelings of isolation (Brown et al., 2003). Being able to participate in the community in a meaningful way and being accepted by others are also important factors for the QOL of young people with a disability (King et al., 2002). For every person it is important to have a sense of belonging and having connections with others, and this is also true for families who have a child with ID/DD (King et al., 2002; Woodill, Renwick, Brown, \& Raphael,1994). A lack of opportunity to participate in community activities influences the QOL of families (Brown et al., 2016). 
International Journal of Child, Youth and Family Studies (2018) 9(4): 107-124

Schippers, Zuna, and Brown (2015) found that families are in continuous interaction with their (social) environments and suggested that micro (the family), meso (community and organisations), and macro elements (policies and government) are all important for understanding the lives of families because the three different levels interact with one another (see Bronfenbrenner, 1976). If we want to understand why families are dissatisfied with their support from others and with community interaction, the continuous interplay between these levels needs to be studied in more detail.

In the Netherlands, it is not just the families who are involved in the care for their family member with ID/DD. Recent political developments that move towards creating a "participation society" mean that society in general has to provide more informal support. The creation of a participation society places a strong requirement on people's informal networks and social communities. Also, the Dutch government wants people with a disability to be included in regular work, instead of in sheltered workplaces (Delsen, 2016; Rijksoverheid, 2013; Verhoeven \& Tonkens, 2013). These ideas have been launched by the government, but practical implementations have only just started.

Given the scientific and societal relevance of the FQOL concept, we have studied the societal factors that influence the FQOL of families in the Netherlands, as part of a larger qualitative study in the Netherlands on FQOL of families who have a child with ID/DD. We feel it is important to take an in-depth look into what factors influence the interactions of families with the community and the support they receive. In this article we therefore focus specifically on the factors that influence support from others and interaction with the community.

\section{Methods}

\section{Study Design}

A qualitative case study of multiple families was undertaken to gain insight into the experiences and needs of families with regard to their FQOL. Case study research is a good strategy to gain in-depth knowledge of a unique and bounded entity in a real-life context (Abma $\&$ Stake, 2014). The family was the unit of analysis, in which the perspectives of multiple family members were included. The families formed a "naturalistic" case study, which means that the families were studied in their daily and normal lives (Abma \& Stake, 2014). We have chosen a multiple case study design (Baxter \& Jack, 2008), because it enabled us to compare the findings across the participating families and also to make a more meaningful contribution to theory (Stake, 2013).

\section{Data Collection}

Qualitative semi-structured interviews were used. In total, 54 family members (33 parents, 14 siblings, and 7 children with ID/DD) were interviewed from 25 families, and 10 people were interviewed twice, those being cases in which the first interview did not cover all 
the topics ( $N=64$ interviews). The main researcher carried out 36 of these interviews, and two undergraduate students and one master's student did the other interviews. Some participants were interviewed together with their spouses, on their request.

We recruited families through schools for special education, because almost all Dutch children with ID/DD are enrolled in such schools. We provided flyers and we also recruited people face-to-face during parent coffee times or meetings in schools. Some parents were also recruited during after-school activities for children with ID/DD. We wanted the families to be as diverse as possible, and our convenience sample did include many types of family - families with one child, multiple children, one parent, more children with an ID, same-sex parents, and families with various cultural backgrounds such as Dutch, Moroccan, and Indo-Surinamese. Demographic characteristics are provided in Tables 1 to 3.

Table 1 Demographic Characteristics of Parents

\begin{tabular}{|c|c|}
\hline Variable & Parents $(n=33)$ \\
\hline Age $(n)$, range (years) & $38-61$ \\
\hline $30-40$ & 3 \\
\hline $41-50$ & 8 \\
\hline $51-60$ & 4 \\
\hline $61-70$ & 2 \\
\hline Missing data & 16 \\
\hline \multicolumn{2}{|l|}{ Gender $(n)$} \\
\hline Female & 23 \\
\hline Male & 10 \\
\hline \multicolumn{2}{|c|}{ Relationship to children ( $n$ ) } \\
\hline Biological mother & 23 \\
\hline Biological father & 10 \\
\hline \multicolumn{2}{|l|}{ Employment (n) } \\
\hline Full-time or part-time & 14 \\
\hline Not working & 10 \\
\hline Missing data & 9 \\
\hline \multicolumn{2}{|l|}{ Cultural origin $(n)$} \\
\hline Dutch & 16 \\
\hline Moroccan & 7 \\
\hline Netherlands Antilles & 1 \\
\hline Indo-Surinamese & 4 \\
\hline \multicolumn{2}{|l|}{ Ethiopian } \\
\hline Pakistani & 2 \\
\hline Nigerian & 1 \\
\hline Missing data & 2 \\
\hline
\end{tabular}

Table 2 Demographic Characteristics of Siblings 
International Journal of Child, Youth and Family Studies (2018) 9(4): 107-124

\begin{tabular}{lc}
\hline \multicolumn{1}{c}{ Variable } & Siblings $(n=14)$ \\
\hline Age $(\boldsymbol{n})$, range (years) & $7-25$ \\
$5-7$ & 1 \\
$8-10$ & 2 \\
$11-13$ & 4 \\
$14-16$ & 2 \\
$17-19$ & 1 \\
$20-22$ & 2 \\
$23-25$ & 2 \\
Gender $(\boldsymbol{n})$ & \\
Female & 10 \\
Male & 4 \\
Position in relation to child with disability & \\
(n) & \\
Older & 10 \\
Younger & 4 \\
Cultural origin $(\boldsymbol{n})$ & \\
$\quad$ Dutch & 6 \\
Moroccan & 6 \\
Indo-Surinamese & 1 \\
Ethiopian & 1 \\
\hline
\end{tabular}

Table 3 Demographic Characteristics of Children with ID/DD

\begin{tabular}{lc}
\hline \multicolumn{1}{c}{ Variable $(n)$} & Children with ID $(n=7)$ \\
\hline Age $(\boldsymbol{n})$, range (years) & $11-22$ \\
$11-13$ & 1 \\
$14-16$ & 2 \\
$17-19$ & 2 \\
$20-22$ & 1 \\
$\quad$ Missing data & 1 \\
Gender $(\boldsymbol{n})$ & \\
Female & 4 \\
$\quad$ Male & 3 \\
Cultural origin $(\boldsymbol{n})$ & \\
$\quad$ Dutch & 5 \\
Moroccan & 1 \\
Indo-Surinamese & 1 \\
\hline
\end{tabular}

During the interviews, a topic list was used to check whether all topics had been covered, but it allowed enough room for the personal stories of the family members. Topics included: daily routines, diagnosis of the ID/DD of the child, relations between family members, things 
International Journal of Child, Youth and Family Studies (2018) 9(4): 107-124

that are important for family life, connections with other people and community, support, personal goals and wishes, and future of the family. With regard to the data on social support and the social environment, we asked general questions about what family members needed in their daily lives, and what things could be improved. These open questions often led to discussions about the presence or lack of support and understanding from others, on which we will elaborate in the Findings section. Also, we checked the FQOLSurvey-2006 and the Beach Center FQOL Scale for specific topics regarding social support and community interaction, which we could use for probing or as a main question during the interviews. The main items regarding social support and the social environment from these two instruments were included in our topic list: what kind of practical and emotional support the family received from friends, (distant) family members, and neighbours; and how the family members experienced their contact with people from their communities (see full topic list in the Appendix). The interviews lasted between 45 and 150 minutes and usually took place in the homes of the families. After receiving consent, all interviews were recorded and transcribed.

\section{Data Analysis}

The interviews and field notes were analysed twice with the principles of inductive thematic analysis (Braun \& Clarke, 2006) and inductive category development (Mayring, 2000). First, for each family, the main researcher extracted all main topics from the interviews and participant observations. After that, the data and quotes were formed into clusters and themes, based on the main topics, that connected the data of all family members within one family. When needed, new topics were formed, or follow-up questions were adjusted. This resulted in a separate family analysis per family. These family analyses were combined in a main frame of analysis with topics and subtopics. Second, all the data were analysed for a second time with the above frame of analysis in mind, and after the final main themes were confirmed and adjusted, subthemes were formed that included part of the transcripts and quotations. The main researcher coded all the material; the three involved students and one other researcher read all the data (each a different part) and checked whether the final interpretations matched. The author team was involved in interpreting the data, a procedure known as inter-rater reliability (Hsieh \& Shannon, 2005). For this article, we focus on findings about the social environment: support, acceptance, understanding, community interaction, and cultural norms and values. Other parts of the analysis that relate to internal family dynamics are discussed elsewhere (see Boelsma, Caubo-Damen, Schippers, Dane, \& Abma, 2017).

\section{Quality Procedures}

To improve the validity of the interviews, a member check was sent to the interviewees to check for inconsistencies and to check whether the interviewees felt their story was correctly represented (Barbour, 2001). Some respondents made minor adjustments to the member check, but otherwise all respondents agreed to the member checks. The data were independently analysed by multiple researchers and the author team to reduce personal bias. Before the start of 
International Journal of Child, Youth and Family Studies (2018) 9(4): 107-124

the research, the research ethics committee of the VU Medical Centre in Amsterdam, which is attached to the Vrije Universiteit Amsterdam [Free University Amsterdam], approved the project.

\section{Findings}

In this section we present our findings relating to the experiences of the families with their social environment and especially with the support they receive. We will illustrate how processes of acceptance and understanding play a role. Also, we will show how the family members experience their interactions with the community, and how moral notions and values are underlying factors. Pseudonyms are used to ensure anonymity.

\section{Support}

Almost all families found support, both emotional and practical, important for their FQOL. Some families described being very closely connected to their extended families, who were available for support when needed. Mother Marieke explained, for example, that when her son Martijn, who has an intellectual disability and autism, was young, her parents were very supportive: "I had a lot of support from my parents. When I came home, they had already given Martijn his meals. My mother would cook, my father would wash and bathe Martijn. My father was like a father to Martijn." Others described how good friends provided emotional and sometimes practical support when it was needed, as father Henk illustrated: "We have two very good friends, they live very close to us, and besides the fact that we are very good friends, they also provide practical support." Some parents worried about the "participation society" in which the social network of people is expected to support families with a child with an ID/DD. Mother Maria explained that her family is trying to support her, but they are not obliged to:

The government wants to make cuts in healthcare, and that is why they want family members to help out. But it is not mandatory, so the participation law just does not work. My sisters both work a lot — everybody has to work. So you cannot expect too much support from them.

While most families received some form of support from family members, there were a few families that had very little support from other people, and this seemed to be connected to the lack of understanding by others of what their family life was like.

\section{Acceptance and Understanding}

Lack of acceptance and understanding seemed to be underlying factors that impeded the support of the social environment, and influenced the FQOL of the families. In some families, the friends, extended family members, and acquaintances withdrew from the family when the son or daughter with an ID/DD was born. Parents explained that sometimes other people acted scared, or did not know how to behave towards the child with an ID/DD. Mother Ans said in this regard, "The first two or three years, we lost contact with more than half of our friends. This was because of a lack of understanding." Anne-Marie, a mother with two daughters with an 
intellectual disability, experienced a lack of understanding from her family: "The family really does not get it at all, they find it especially difficult to understand the behaviour of Roos." Her third daughter Mila found that her current friends are sensitive towards her situation at home, but at her secondary school she felt this had been very different: "I remember that in secondary school, people really did not get it. They did not understand what it meant to have an intellectual disability, and that having my sisters around affected my home situation."

Family members observed that they often experienced a lack of understanding and acceptance in their interactions with other people in their communities. For example, Father Ed explained what happened when he took his daughter Janet, who had Down syndrome, to a regular primary school ${ }^{1}$ for the first time:

People were scared, it is something they do not know: "Our children in the class with a child with an ID, that is not possible!" And the worst thing is: they were jealous because they thought my daughter would withdraw attention from others in the class. Once they got to know her, everybody loved her.

Some parents explained that they felt their employers were supportive and flexible in case of an emergency at home, but other parents felt that their colleagues were unsupportive and showed a lack of understanding, as Anne-Marie explained: "I get sick of colleagues saying: you only have half a job. Then I think, well, when I get home I have the other half! They just do not understand."

Besides feeling understood, acceptance was also important according to the family members. Father Bas, who has an ID like his son Jack, explained that when Jack had to go to special education, he lost his friends in the neighbourhood: "They don't accept him anymore. He had a lot of friends here at the regular primary school, but when he had to go to special education he lost them all. Very strange." Jack confirmed this: "They bully me. When I had to leave the regular school, they suddenly were not friends of mine any more. Because I was special."

\section{Community Interaction}

Often, it is not immediately visible to strangers that a child has an ID/DD. Many family members found this difficult, because other people might have expectations of the child that cannot be met. Father Theo, for example, said, "Because Jesse looks normal at first sight, people expect him to behave in socially accepted ways." Another father, Piet, said at first that he did not think people treated their family differently because of their son Martin with an ID, who is 15 years old. Martin was able to participate in traffic with his bike, but Piet noticed that sometimes people gave strange looks because he acted differently in traffic than did other adolescents. Quite a few parents said that they wished their child had a visible intellectual disability like Down

\footnotetext{
${ }^{1}$ In the Netherlands, children with an ID usually go to schools for special education. After a couple of years of regular education, Janet transferred to such a school.
} 
International Journal of Child, Youth and Family Studies (2018) 9(4): 107-124

syndrome, so other people would know that the child had an ID and behaved differently than other children. Also the children with ID/DD themselves sometimes felt unable to meet expectations from others in their interactions in the community. Maria, an adolescent with an ID, was afraid to travel by bus because she was afraid that people would ask her things and she would not know what to say. Also, at times the interactions with strangers led to funny and positive situations, as sister Anis illustrated when she talked about her younger brother with ID:

When we are in the bus, he will just talk to anyone, and when people talk back he has that immediate connection with them. I remember one time when he talked to a woman in the bus, and when she got out of the bus he gave her a hug, and everyone in the bus was like, "Ah, how sweet!"

\section{Societal Norms and Values}

\section{Case Example}

Parents Sanne (63 years old) and Theo (62 years old) have two children: son Tom (21 years old) with an ID and daughter Ingrid (23 years old). Both parents have a high education and above-average income. Sanne and Theo explained that when Tom was young, they unconsciously tried to make him as "normal" as possible. Sanne said: "I believe that I was someone who, when he was a little boy, was very busy with how to make him as normal as possible. How to use all of his talents, and that deep down you hope that a day will come that he will become a normal person. Even though you know, of course, when you get the diagnosis, that that is not going to happen. And when I think back on it, I wonder if we did the right thing." Her husband Theo continued: "We had a lot of talks about that: how normal do you want him to be." Sanne said that this is partly because of the expectations society has: "It is an imposed idea: that a normal person is a valuable person. In that sense these are societal expectations, also from your own upbringing, that a normal person can participate in society." Theo experienced these societal rules and expectations also in the social education of his son: "One time at the school for special education, we were told that we had not raised Tom well because he did not use the polite form of 'you' when he talked to people. He clearly has no idea of what that means." Their daughter Ingrid sometimes goes shopping with Tom, and she found that people often look at them funny: "Because Tom looks quite normal, people think, 'He is acting weird'. The other day I went to buy shoes with him, and that is always very difficult because he is very sensitive. He feels every little bump in the shoes. And he also has a small size for a man, so that is difficult. I am used to him trying on a shoe for 10 minutes, but every time the shopkeepers really don't get it, so I need to tell them, 'Just come back in 10 minutes, he needs more time to try it on.' Luckily this time Tom did not make a scene, but this is a situation in which you know that people are thinking, 'What an odd couple!' But I just don't care."

Various family members felt that the social community has certain norms and values that people are expected to live up to. Family members sometimes felt stared at or frowned upon in 
International Journal of Child, Youth and Family Studies (2018) 9(4): 107-124

their interactions with others. Sister Samira said that her brother Nabil evoked such reactions from other people: "In the outside world people can look like: 'Who is that?', and 'Why is he like that?' "Jack, who has an ID, said he felt like everyone else, but that other people treated him as being different. Families sometimes had trouble "fitting in", and some recalled that at times they had tried to be a "normal" family like others, until they realized that this was unrealistic and undesirable. Family members also felt that their "special" family characteristics had enriched them: parents and siblings, for example, felt that they experienced a more diverse perspective on life than many other people, and some had found talents in themselves that they otherwise would not have found.

Parents with a non-Dutch background sometimes felt pressure from specific social expectations in their culture that may differ from the Dutch culture. A few mothers with Moroccan roots, for example, explained that in Moroccan culture family members are expected to visit one another on a regular basis. Families with children with an ID/DD found that they could not always meet those expectations because they lived a different family life. Also, it is common in some cultures that the father is the one who takes care of the financial needs of the family, and the mother is more involved in household chores and caretaking. In families with a child needing more attention and care, fathers often took up more household chores. Other family members sometimes found this hard to understand, and mother Hathisa explained, for example:

When I could not do it, my husband did everything at home. When I had my eldest daughter, we went on holiday to his parents in Morocco. And my husband would carry my daughter and change her diapers. Well, they looked at him as if he was from Mars: "You do not do that! Your wife is supposed to do that!" And he said, "Why? She is as much my daughter."

Societal developments, which sometimes inhibit certain cultural norms and values, also influenced the lives of the families. Some parents expressed their concern about new technological developments like non-invasive prenatal testing (NIPT) which can detect Down syndrome at an early stage of pregnancy. Anne-Marie said in this regard:

I think that having a child with Down syndrome, with all the technological means that are available to detect and prevent it, is not something that is welcomed by society. I think that we are moving towards a society in which children who are predicted to have a certain disability are not welcome any more.

Father Ed explained that Anne-Marie and he knew that their daughter Tess would be born with Down syndrome. Ed said, "I still meet people who tell me, 'I don't understand that you chose to have Tess'. Even though she is such a sweetheart. The society thinks, these children are expensive. Yes, so what? They have no right to life?" 
International Journal of Child, Youth and Family Studies (2018) 9(4): 107-124

Our findings may indicate that moral norms and values in society (e.g., about "normality", socially accepted behaviour, and cultural norms about gender roles and family relationships) are reflected in the way the community accepts and understands the families. It might be difficult for other people to understand and accept that "special" families deviate from these societal norms. In turn, this lack of acceptance and understanding might affect how the social community is willing to be involved in supporting and interacting with the families.

\section{Discussion}

In this study we found that a lack of acceptance and understanding can be impeding factors for the FQOL of the participating families. These factors could also contribute to experiences of having a lack of social support. Because of a lack of understanding, connections with friends and extended family members are sometimes lost. Moreover, families reflect on notions of normality and being different, and sometimes struggle with the implicit norms and values of society, which their child with ID/DD and their family cannot always live up to.

Davis (1997/2006) indicated that "we live in a world of norms" (p. 3), and argued that the concept of "normalcy" is of recent origin, arising in part from the rapid development of statistics in the early 19th century: the current meaning of the word "normal" entered the English language only in 1840. The concept allows the ranking by society of desirable and undesirable characteristics of people, and the classification of people as "normal" or "abnormal". Statistics made available the calculation of the arithmetic mean, which could be used to measure the extent to which a characteristic deviated from this norm (Davis, 1997/2006).

The families in our study felt confronted by these norms in their daily lives through their interactions with others. Our findings have some resemblance to studies on parents with children with autism, who often feel isolated from "normal" life (Gray, 1993, 1997; Woodgate, Ateah, \& Secco, 2008). Woodgate and colleagues (2008) found that this was partly due to a lack of understanding shown by extended family members. However, Gray (1997) found that parents perceive themselves as not being able to live a normal life due to their families, while in our study the families felt that it was also the social environment that imposed these norms of normality. One could argue that "normality" is a myth, and that the variations between people actually enrich society. Along the same lines, Barnes and Mercer (2001) advocated the concept of "disability culture", a culture in which the norm is questioned and actions are undertaken to celebrate the diversity of people.

Studies on community participation and social inclusion of people with an ID/DD have found that acceptance is indeed one of the important factors in interaction with the community (Abbott \& McConkey, 2006; Cobigo et al., 2016; Hall, 2009; Link \& Phelan, 2001). However, these studies include the perspectives of the main caregiver and/or adults with an ID/DD, but not the perspectives of additional family members. Our study shows that interaction with the community is relevant to the quality of families' lives. Family members experience feelings of 
International Journal of Child, Youth and Family Studies (2018) 9(4): 107-124

not being a "normal" family; and sometimes they feel criticised by the social environment and experience a lack of social integration of their family. Acceptance and understanding of others for their situation are important for the social inclusion of families, and this is especially the case in a society in which people are expected to support others in their community, like the "participation society" that the Dutch government is striving for.

The two well-known FQOL instruments, the Beach Center FQOL Scale and the FQOL Survey-2006, have found that social support and community interactions are scored low by families; however, these instruments do not include items about acceptance and understanding of others, which could be underlying processes that are a prerequisite for support and social participation. It might therefore be valuable to include these topics in (qualitative) follow-up interviews or studies. Acceptance and understanding are important factors to consider when understanding FQOL because they provide additional contextual information about the families.

Community interaction, which is also generally scored low by families, is part of the FQOLSurvey-2006; the domain focuses on the effort families take to participate in society and what options are available for them to do so. These indicators are broadly formulated, but do not zoom in on whether people and families feel accepted by society and if, for example, they feel that they are perceived as "normal". In surveys, respondents can expand on this in the open field provided within the instrument, but few systematic reports about these qualitative data have been made. It would be very valuable if researchers could expand and reflect on these additional data more often.

\section{Limitations}

A limitation of this study is that the findings with regard to the social context and cultural values and norms are specific to the Netherlands. It is possible that the norms of Dutch society differ in relevant ways from those of other countries. At the same time, we expect that families in other countries may have similar experiences of their interactions with the community and their support from people. Another limitation is that the data from children with an ID/DD were less substantial than the data from their parents and siblings with regard to their perspectives on support and their social environment. Also, the use of multiple interviewers could be a limitation because there might be small variations in the use of the topic list and probing by the different researchers.

\section{Conclusions and Implications}

Our study shows that it is important to consider that societal factors influence the lives of families. We have focused on the perspectives of the different family members, but in follow-up research it would be interesting to study the social environment of families as well - extended family members, friends, neighbours, professionals, and others - to get a better idea of their perspectives and more insight into how inherent norms of normality are mechanisms in the social and support contacts between people and how these are linked to the FQOL of families. 
Second, our study shows that a lack of acceptance and understanding concerns not only the person with an ID/DD, but affects the whole family in their daily interactions with people and the support they receive. It is important to include the perspectives of family members with regard to their social inclusion: Do they feel accepted and understood as a family? What kind of processes of inclusion and exclusion do they encounter in their daily family life? And how do those processes influence the support received by the family and their feelings of belonging to society? We would recommend that care workers and other professionals that work with families be sensitive to these issues.

When evaluating the QOL of families, it can be useful to use an FQOL instrument. The two existing FQOL instruments both have the goal of evaluating services and support for families, focusing on both emotional and practical support. In the Netherlands, support will devolve more and more onto the informal network of people, supplementing professional support, and this calls for an evaluative instrument that is sensitive to the underlying processes that inhibit or foster the extent to which the social networks of families are inclined to provide support. Changing the focus of support may prove difficult, because it is not only professional institutions that need to be receptive to change, but also the community. In this line, our findings might also be relevant for professionals that work with other disadvantaged groups struggling to meet the dominant societal norms about normality, such as families with a family member with a different disability or mental illness, single parents, or LGBT parents. They may encounter similar problems with regard to understanding, acceptance, and support, which in turn could influence their FQOL.

We learned from our study that acceptance and understanding could be important underlying factors for the support that families with a child with an ID receive. Also, implicit and explicit cultural norms and values could potentially influence the way society includes and supports people with an ID/DD and their families. More awareness and research on these topics could help to improve available FQOL instruments and the lives of families. 
International Journal of Child, Youth and Family Studies (2018) 9(4): 107-124

\section{References}

Abbott, S., \& McConkey, R. (2006). The barriers to social inclusion as perceived by people with intellectual disabilities. Journal of Intellectual Disabilities, 10(3), 275-287. doi: $10.1177 / 1744629506067618$

Abma, T. A., \& Stake, R. E. (2014). Science of the particular: An advocacy for naturalistic case study in health research. Qualitative Health Research, 24(8) 1150-1161. doi: $10.1177 / 1049732314543196$

Barbour, R. S. (2001). Checklists for improving rigour in qualitative research: A case of the tail wagging the dog? The BMJ, 322, 1115-1117. doi: 10.1136/bmj.322.7294.1115

Barnes, C., \& Mercer, G. (2001). Disability culture: Assimilation or Inclusion. In G. L. Albrecht, K. Seelman, \& M. Bury (Eds.), Handbook of disability studies (pp. 515-534). Thousand Oaks, CA: Sage.

Baxter, P., \& Jack, S. (2008). Qualitative case study methodology: Study design and implementation for novice researchers. The Qualitative Report, 13(4), 544-559.

Beach Center on Disability. (2005). The Beach Center Family Quality of Life Scale. Lawrence, KS: Beach Center, University of Kansas, in partnership with families, service providers, and researchers.

Boelsma, F., Caubo-Damen, I., Schippers, A., Dane, M., \& Abma, T. A. (2017). Rethinking FQoL: The dynamic interplay between individual and family quality of life. Journal of Policy and Practice in Intellectual Disabilities, 14(1), 31-38. doi:10.1111/jppi.12224

Braun, V., \& Clarke, V. (2006). Using thematic analysis in psychology. Qualitative Research in Psychology, 3(2), 77-101. doi:10.1191/1478088706qp063oa

Bronfenbrenner, U. (1976). The ecology of systems. Cambridge, MA: MIT Press.

Brown, I., Anand, S., Fung, W.A. L., Isaacs, B., \& Baum, N. (2003). Family quality of life: Canadian results from an international study. Journal of Developmental and Physical Disabilities, 15(3), 207-230. doi:10.1023/A:1024931022773

Brown, I., Brown, R. I., Baum, N. T., Isaacs, B. J., Myerscough, T., Neikrug, S., ... \& Wang, M. (2006). Family Quality of Life Survey: Main caregivers of people with intellectual disabilities. Toronto, ON: Surrey Place Centre.

Brown, R. I., Kyrkou, M. R., \& Samuel, P. S. (2016). Family quality of life. In I. L. Rubin, J. Merrick, D. E. Greydanus, \& D. R. Patel (Eds.), Health care for people with intellectual and developmental disabilities across the lifespan (pp. 2065-2082). New York, NY: Springer International. 
International Journal of Child, Youth and Family Studies (2018) 9(4): 107-124

Cobigo, V., Brown, R., Lachapelle, Y., Lysaght, R., Martin, L., Ouellette-Kuntz, H., \& Fulford, C. (2016). Social inclusion: A proposed framework to inform policy and service outcomes evaluation. Inclusion, 4(4), 226-238. doi:10.1352/2326-6988-4.4.226

Davis, L. J. (2006). Constructing normalcy: The bell curve, the novel, and the invention of the disabled body in the nineteenth century. In L. J. Davis (Ed.), The disability studies reader (2nd ed., pp. 3-16). New York, NY; Taylor \& Francis. (Original work published 1997)

Delsen, L. (2016). The realisation of the participation society. Welfare state reform in the Netherlands: 2010-2015 (Working paper NiCE16-02). Nimeguen, The Netherlands: Institute for Management Research, Radboud University. Retrieved from http://www.ru.nl/publish/pages/516298/nice_16-02.pdf

Faragher, R., Broadbent, C., Brown, R. I., \& Burgess, J. (2014). Applying the principles of quality of life to education. In R. I. Brown \& R. M. Faragher (Eds.), Quality of life and intellectual disability: Knowledge application to other social and educational challenges (pp. 37-54). New York, NY: Nova.

Gray, D. E. (1993). Perceptions of stigma: The parents of autistic children. Sociology of Health \& Illness, 15(1), 102-120. doi:10.1111/1467-9566.ep11343802

Gray, D. E. (1997). High functioning autistic children and the construction of "normal family life". Social Science \& Medicine, 44(8), 1097-1106. doi:10.1016/S0277-9536(96)00237-7

Hall, S. A. (2009). The social inclusion of people with disabilities: A qualitative meta-analysis. Journal of Ethnographic \& Qualitative Research, 3(3), 162-173.

Hoffman, L., Marquis, J., Poston, D., Summers, J. A., \& Turnbull, A. (2006). Assessing family outcomes: Psychometric evaluation of the Beach Center Family Quality of Life Scale. Journal of Marriage and Family, 68(4), 1069-1083. doi:10.1111/j.1741-3737.2006.00314.x

Hsieh, H.-F., \& Shannon, S. E. (2005). Three approaches to qualitative content analysis. Qualitative Health Research, 15, 1277-1288. doi:10.1177/1049732305276687

IASSID SIRG-QOL (International Association for the Scientific Study of Intellectual Disabilities Special Interest Research Group on Quality of Life). (2000). Quality of life: Its conceptualization, measurement, and application. A consensus document. Retrieved from https://www.iassidd.org/content/QOL Consensus Document 2000

King, G., Tucker, M. A., Baldwin, P., Lowry, K., Laporta, J., \& Martens, L. (2002). A life needs model of pediatric service delivery: Services to support community participation and quality of life for children and youth with disabilities. Physical \& Occupational Therapy in Pediatrics, 22(2), 53-77. doi:10.1080/J006v22n02_04 
International Journal of Child, Youth and Family Studies (2018) 9(4): 107-124

Link, B. G., \& Phelan, J. C. (2001). Conceptualizing stigma. Annual Review of Sociology, 27(1), 363-385. doi:10.1146/annurev.soc.27.1.363

Mayring, P. (2000). Qualitative content analysis [28 paragraphs]. Forum Qualitative Sozialforschung / Forum: Qualitative Social Research, 1(2), Art. 20. Retrieved from http://nbn-resolving.de/urn:nbn:de:0114-fqs0002204

Poston, D., Turnbull, A., Park, J., Mannan, H., Marquis, J., \& Wang, M. (2003). Family quality of life: A qualitative inquiry. Mental Retardation, 41(5), 313-328. doi:10.1352/00476765(2003)41<313:FQOLAQ>2.0.CO;2

Rijksoverheid [National Government of the Netherlands]. (2013). Troonrede [Throne speech]. Retrieved from https://www.rijksoverheid.nl/documenten/toespraken/2013/09/17/troonrede$\underline{2013}$

Schalock, R. L., Brown, I., Brown, R., Cummins, R. A., Felce, D., Matikka, L, ... \& Parmenter, T. (2002). Conceptualization, measurement, and application of quality of life for persons with intellectual disabilities: Report of an international panel of experts. Mental Retardation, 40(6), 457-470. doi:10.1352/00476765(2002)040<0457:CMAAOQ>2.0.CO;2

Schippers, A., Zuna, N., \& Brown, I. (2015). A proposed framework for an integrated process of improving quality of life. Journal of Policy and Practice in Intellectual Disabilities, 12(3), 151-161. doi:10.1111/jppi.12111

Stake, R. E. (2013). Multiple case study analysis. New York, NY: Guilford Press.

Verhoeven, I., \& Tonkens, E. (2013). Talking active citizenship: Framing welfare state reform in England and the Netherlands. Social Policy and Society, 12(03), 415-426. doi:10.1017/S1474746413000158

Woodgate, R. L., Ateah, C., \& Secco, L. (2008). Living in a world of our own: The experience of parents who have a child with autism. Qualitative Health Research, 18(8), 1075-1083. doi: $10.1177 / 1049732308320112$

Woodill, G., Renwick, R., Brown, I., \& Raphael, D. (1994). Being, belonging, becoming: An approach to the quality of life of persons with developmental disabilities. In D. Goode (Ed.), Quality of life for persons with disabilities: International perspectives and issues (pp. 5774). Cambridge, MA: Brookline Books.

Zuna, N. I., Brown, I., \& Brown, R. I. (2014). Family quality of life in intellectual and developmental disabilities: A support-based framework. International Public Health Journal, 6(2), 161-184. 
International Journal of Child, Youth and Family Studies (2018) 9(4): 107-124

\section{Appendix}

\section{Interview Topic List}

\section{Background Information}

1. Could you tell us something about yourself and your family (work, hobbies, personal contacts)?

2. Could you describe a typical weekday for your family? And what kinds of things happen during the week (routines, daily occurrences, school, work, care, moments together)?

3. Could you tell us something more about your children, and the disability of your son/daughter (diagnosis, reaction from the family and the social environment, changes in the family after child with ID/DD was born, expectations for the future)?

4. Could you describe the relationships between the family members?

5. What kind of contacts between the family and their social environment are there, and what are your experiences with these contacts (friends, families, organisations, neighbourhood, how do these contacts influence the well-being of the family, what would you like to see changed with regard to contacts with the social environment)?

\section{Family Quality of Life}

6. What comes to mind when you think about the things your family needs in daily life? What things are important for your family? (Draw mindmap.)

7. Can you tell us something more about these important things and what would you like to see different?

8. Could you describe what things impact your family life in a negative or positive way?

9. What tasks are assigned to the different family members? How do you experience your own role in the family?

10. Does your family receive support from others, or professionals? If so, what kind of support, how satisfied are you, and how important is this support for the family?

11. How do the individual needs in the family relate to the needs of the family as a whole?

12. Did having a child with ID/DD impact the possibilities of a personal career of (one of) the parents?

13. How do you see the future of your family? 
International Journal of Child, Youth and Family Studies (2018) 9(4): 107-124

\section{Individual Quality of Life}

14. What things are important for you to have a good and fulfilling life?

15. How do you experience your own personal life at the moment? What are the things you are (not) satisfied with?

16. At what moments do your family circumstances influence your own personal well-being?

17. When you think about the best moments with the family, what comes to mind? 